\title{
Diagnosis of Fibrotic Distal Ileum Stenosis after Ischemic Enteritis Using Transabdominal Ultrasonography
}

\author{
Ryo Katsumata $^{a}$ Noriaki Manabe ${ }^{a}$ Masaki Matsubara ${ }^{b} \quad J u n$ Nakamurac \\ Kazuma Kawahito ${ }^{a}$ Maki Ayaki $^{a}$ Minoru Fujita ${ }^{a}$ Aya Sunago ${ }^{d}$ \\ Hideyo Fujiwara ${ }^{e}$ Yasumasa Monobe ${ }^{e}$ Tomoari Kamadad \\ Hirofumi Kawamotoc ${ }^{c}$ Tomoki Yamatsuji ${ }^{b}$ Yoshio Naomoto ${ }^{b}$ \\ Ken Haruma ${ }^{c}$ \\ aDepartment of Clinical Pathology and Laboratory Medicine, Kawasaki Medical School \\ General Medical Center, Okayama, Japan; 'bepartment of General Surgery, Kawasaki \\ Medical School General Medical Center, Okayama, Japan; 'Department of General \\ Internal Medicine 2, Kawasaki Medical School General Medical Center, Okayama, Japan; \\ dDepartment of Health Care Medicine, Kawasaki Medical School General Medical Center, \\ Okayama, Japan; e Department of Pathology, Kawasaki Medical School General Medical \\ Center, Okayama, Japan
}

\section{Keywords}

Ischemia · Enteritis · Renal dialysis · Ultrasonography

\begin{abstract}
Ischemic enteritis (IE) is a rare disorder which is caused by inadequate blood flow to small intestine. The diagnostic procedure of this disease has not sufficiently established because of its rarity. Here, we report a case of IE in a hemodialysis-dependent 70-year-old man and summarize the diagnostic options for IE. The patient was admitted to our hospital because of acute abdominal distention and vomiting. He presented with mild tenderness in the lower abdomen and slightly elevated C-reactive protein level as revealed by blood tests. Radiographic imaging showed small bowel obstruction due to a stricture in the distal ileum. Contrast-enhanced abdominal ultrasonography revealed a $7-\mathrm{cm}$ stenotic site with increased intestinal wall thickening, which preserved mucosal blood perfusion. Elastography revealed a highly elastic alteration of the stenotic lesion, indicating benign fibrotic changes resulting from chronic insufficient blood flow. Based on a clinical diagnosis of IE with fibrous stenosis, a partial ileostomy was performed. After surgical treatment, oral intake was initiated without recurrence of intestinal obstruction. Pathological findings revealed deep ulceration with inflammatory cell infiltration at the stenotic site. Occlusion and hyalinization of the venules in the submucosal layer indicated IE. In addition to current case, we reviewed past case reports
\end{abstract}


of IE. Through this case presentation and literature review, we summarize the usefulness and safety of transabdominal ultrasonography for diagnosing IE.

\section{Introduction}

Ischemic enteritis (IE) is an acute or chronic condition that results from insufficient blood perfusion in the small intestine. Known risk factors of IE include renal failure, arteriosclerosis, chronic heart failure, and diabetes mellitus $[1,2]$. In contrast to ischemic colitis, which results from inadequate blood flow in the large intestine, most IE patients progress to a severe condition requiring operative intervention [1]. Due to the low prevalence of the disease and difficulty in diagnosis, only a few case reports related to this disease have been published till date. Several diagnostic methods, including angiography, balloon-assisted endoscopy, and capsule endoscopy, have been suggested to diagnose IE [2-4].

At the early clinical stage, patients with IE show nonspecific abdominal symptoms, such as mild tenderness and distention. In some cases, IE causes fibrous stenosis resulting in intestinal obstruction $[1,3,5]$. Thus, differential diagnoses range widely from benign to malignant [1]. Although several investigations can be performed to detect IE, there is no gold standard for its diagnosis. Here, we report the first case of IE with fibrous stenosis in the ileum that was diagnosed using transabdominal ultrasonography (TUS). Moreover, we reviewed previously published case reports of IE. Regarding literature collection, we searched for case reports of IE using the following terms on PubMed/MEDLINE ("ischemic enteritis" or "ischemic ileitis") AND ("case" or "case report") by the end of February 2021. The inclusion criteria were case reports with the clinical course from admission to treatment and full articles with abstracts and reports written in English.

\section{Case Report/Case Presentation}

A 70-year-old man was referred to our institution because of abdominal distention and vomiting that started 2 days prior to admission. The patient was undergoing hemodialysis for chronic nephritis, and his past history included hypertension, chronic heart failure, and arterial sclerosis. He was never a smoker and had no history of abdominal surgery or nonsteroidal anti-inflammatory drug use. Upon hospitalization, the patient's blood pressure was 104/57 $\mathrm{mm} \mathrm{Hg}$, heart rate was 78 beats/min, and body temperature was $36.5^{\circ} \mathrm{C}$. Physical examination revealed mild tenderness and swelling in the lower abdomen; the bowel sounds were enhanced. No purpura was found, which is characteristic of Henoch-Schönlein purpura.

A blood test performed on admission revealed mild anemia (hemoglobin $11.2 \mathrm{~g} / \mathrm{dL}$ ), hypoalbuminemia $(2.6 \mathrm{~g} / \mathrm{dL})$, mildly elevated C-reactive protein $(3.24 \mathrm{mg} / \mathrm{dL})$, and elevated serum creatinine $(5.24 \mathrm{mg} / \mathrm{dL})$ levels. The stool culture test results were negative. Tuberculosis and anisakiasis were ruled out by interferon-gamma releasing assays and anisakis antibody tests, respectively.

Abdominal radiography in the standing position revealed dilatation of the small intestine and air-fluid levels in the intestine (shown in Fig. 1a); plain computed tomography (CT) showed a stenotic site with wall thickening and dilatation of the small intestine on the oral side from the stenosis (shown in Fig. 1b, c). After admission, we inserted an 

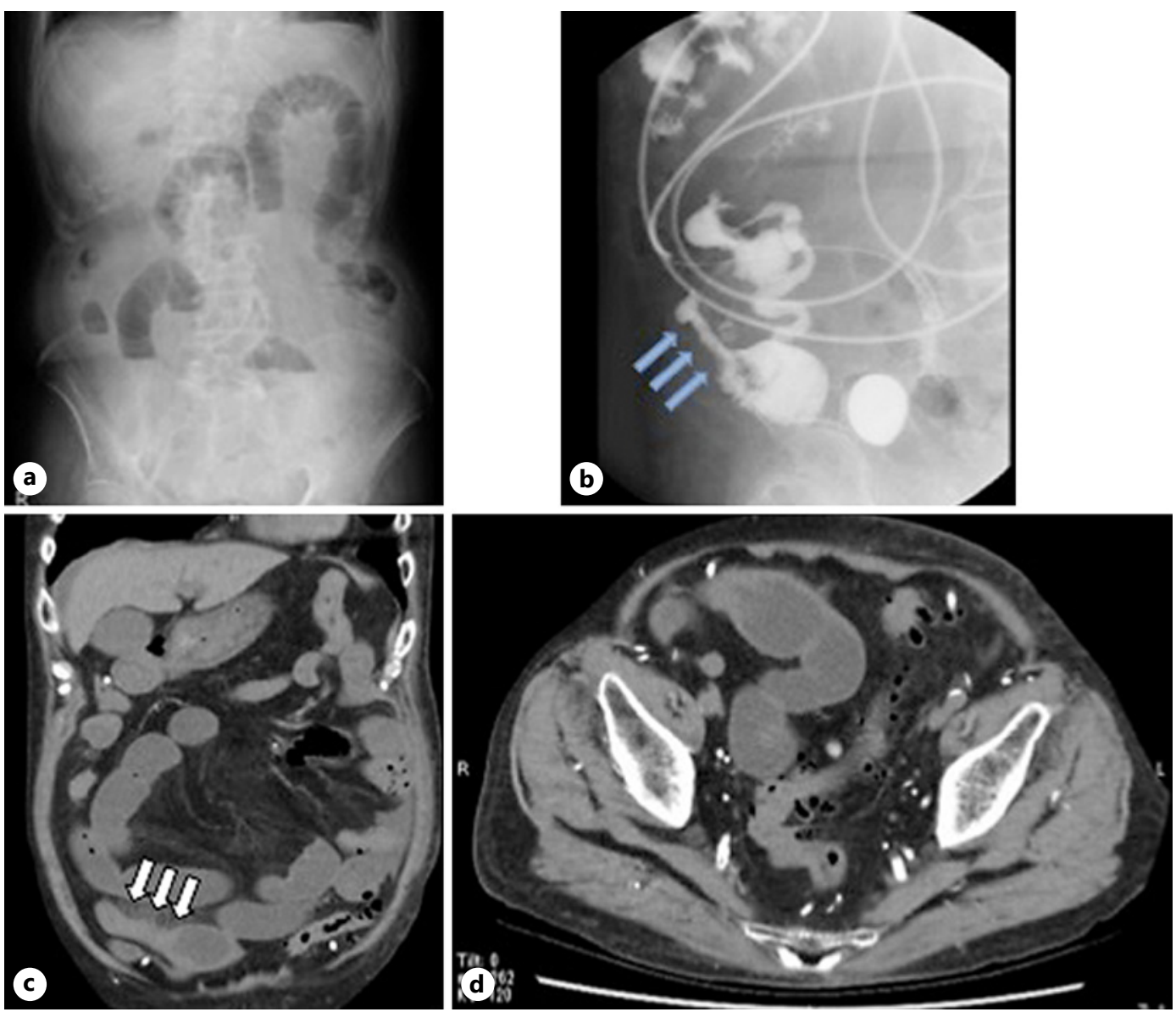

Fig. 1. a Abdominal radiography reveals dilatation of the small intestine and presence of air-fluid levels in the standing position. b, c Abdominal plain CT reveals intestinal wall thickening and a stricture at the distal ileum (white arrows). Dilation of the small intestine detected on the oral side from the stenotic site. Coronal image (a) axial image (b). d Small intestine series demonstrates a tubular stricture at the end of the ileum (blue arrows). CT, computed tomography.

ileus tube to ameliorate the elevated intraluminal pressure. Contrast medium from the tube showed a stricture at the end of the ileum (shown in Fig. 1d). We conducted TUS using Sonazoid TM and elastography techniques, which demonstrated a thickened wall and unclear stratification of the intestinal wall in the lower right abdomen (shown in Fig. 2a, b). The length of stricture was $70 \mathrm{~mm}$, and the minimum lumen diameter was $5 \mathrm{~mm}$. The small intestine on the oral side of the lesion was markedly dilated. Contrast-enhanced ultrasonography showed sufficient mucosal blood flow in the stenotic lesion (Fig. 2c). Ultrasound elastography revealed lower elasticity of the intestinal lesion site than the elasticity of other intact parts of the small intestine that did not show wall thickening or stenosis (strain ratio [local deformation ratio under slight pressure]: 2.52) (Fig. 2d). Based on these findings, the patient was clinically diagnosed with IE. Conservative therapies including fasting and total parenteral nutrition were initiated after the diagnosis; however, the patient's condition did not improve.

Surgery was performed, and the stenotic lesion was surgically resected. Macroscopic findings of the resected ileum demonstrated circumferential ulceration and stricture. Microscopic findings demonstrated penetration of the surrounding tissue and infiltration of inflammatory cells mainly in the mucosal layer. The venules in the submucosal layer 


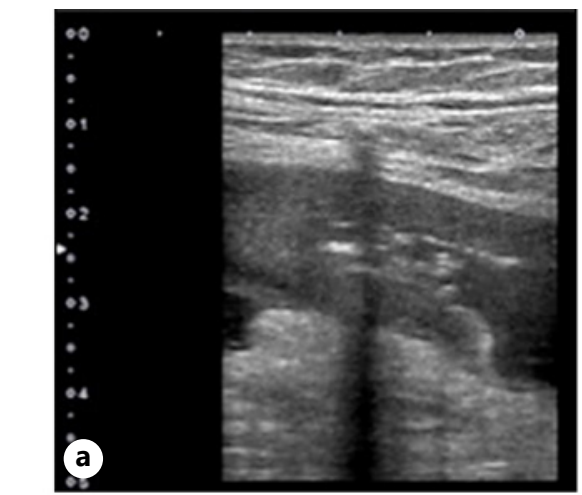

Katsumata et al.: Diagnosis of Fibrotic Distal Ileum Stenosis after Ischemic Enteritis
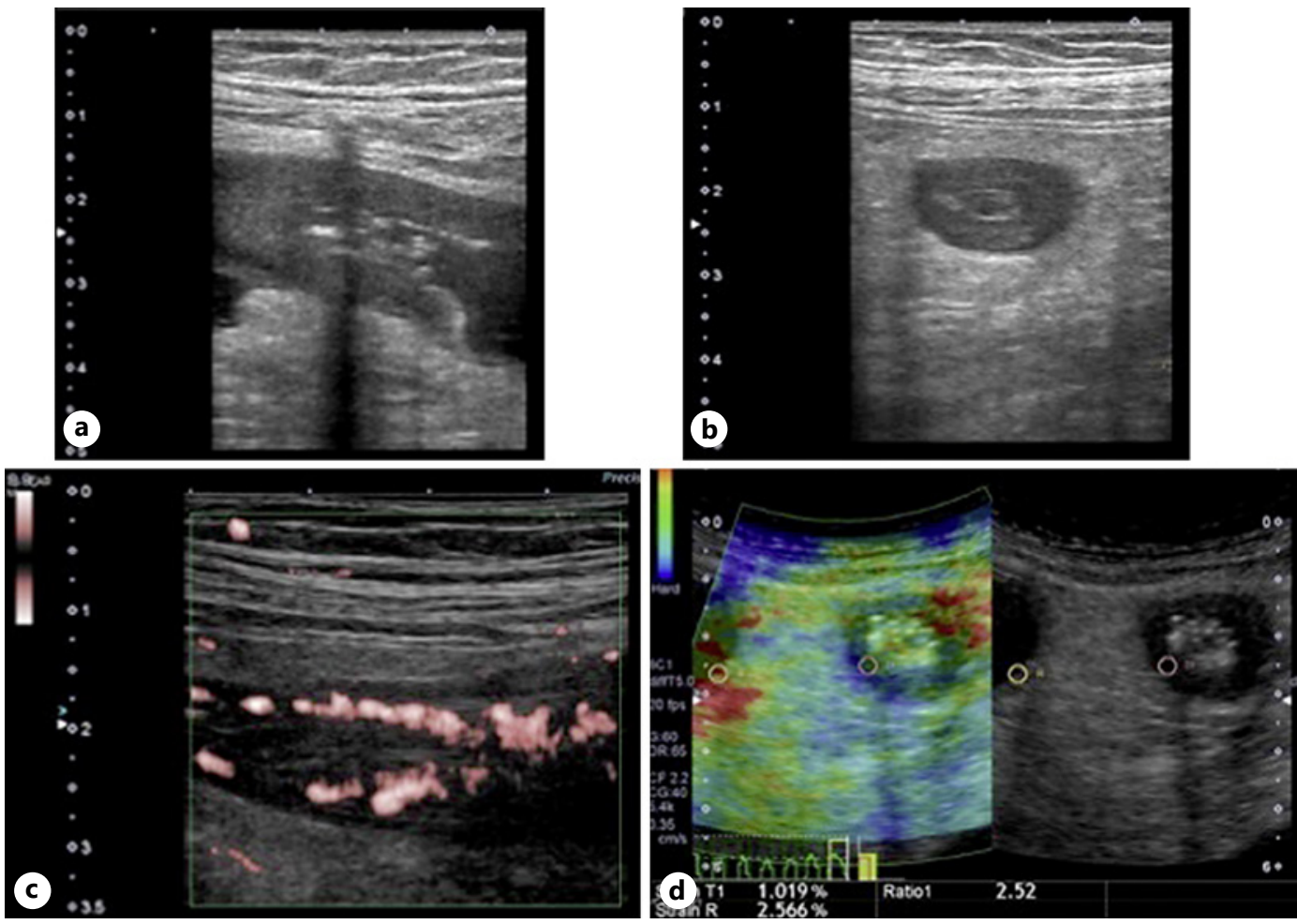

Fig. 2. a, b The B (brightness) mode of TUS illustrates longitudinal wall thickening and a circular stricture at the distal ileum. c Contrast-enhanced ultrasonography demonstrates adequate mucosal blood flow in the stenotic lesion. The length of stricture was $70 \mathrm{~mm}$. d Ultrasound elastography reveals higher elasticity at the stenosis site than the un-thickened small intestine. TUS, transabdominal ultrasonography.

were occluded by hyalinization (shown in Fig. 3a-d). Two days after the operation, the patient started oral intake without the recurrence of IE and was discharged from our hospital 1 month later. There was no relapse when the patient was last assessed 1 year postoperatively.

\section{Discussion/Conclusion}

To the best of our knowledge, this is the first English case report of IE delineated by TUS. Since the prevalence of IE is quite low and it is difficult to diagnose, only a limited number of case reports concerning this condition have been published in English so far [1-7]. A summary of the clinical backgrounds, relevant diagnostic methods, treatment, and outcomes from previous case reports is presented in Table 1. Our patient was an elderly man, as is typical for IE, and his symptoms and clinical course were largely consistent with those of the previous reports.

IE is thought to share risk factors with other ischemic diseases, such as myocardial infarction and stroke [2]. Among the well-known risk factors for ischemic disease, the patient displayed hypertension and hemodialysis. Hemodialysis is a risk factor for ischemic conditions in the gastrointestinal tract, including the small intestine [8]. Repeated hypotensive episodes during hemodialysis elevate the risk of ischemia of the mesenteric artery [9]. Additionally, the prevalence of peripheral arterial disease (a relevant risk factor of IE) among hemodialysis patients is reportedly higher than that in the general population and is $24 \%$ among hemodialysis patients and $4.3 \%$ in the general population [10]. For patients aged $>60$ 

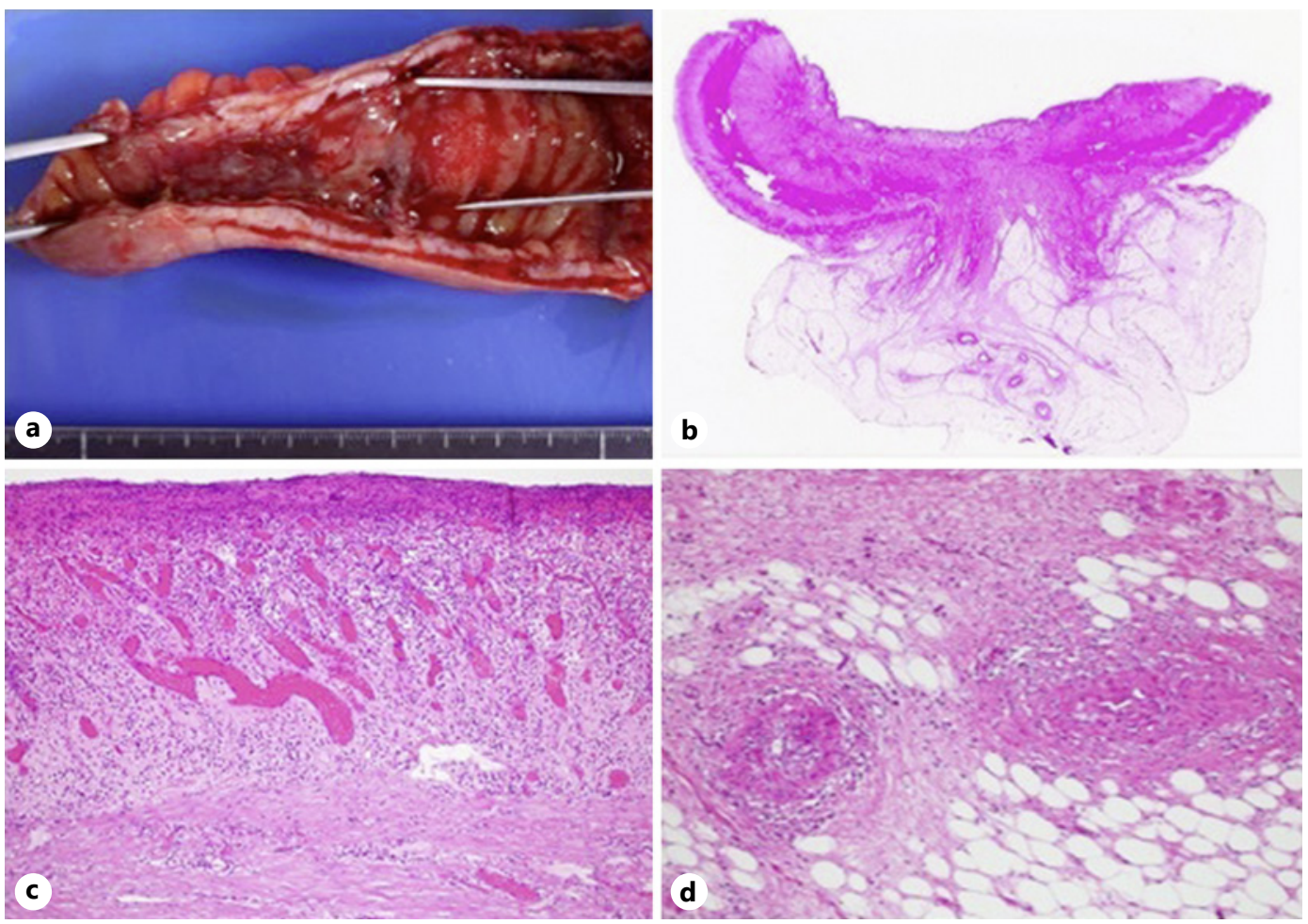

Fig. 3. a Macroscopic findings of the resected specimen demonstrate circumferential ulceration with stenosis. b Microscopic findings reveal a UI-IV ulcer with penetration to the peripheral lipid tissue. c Loss of crypt and inflammatory cell infiltrate. d Dilatation and congestion of submucosal venules (b-d: H\&E staining, c, d: $\times 40$ ) UI, ulcer index; H\&E, hematoxylin and eosin.

years, hemodialysis and hypertension were found to be associated with the onset of ischemic colitis [11]; hemodialysis in particular was associated with high mortality rates [12]. Based on this information, although only a few case reports of IE with hemodialysis have been published, patients undergoing hemodialysis (as in our case) are considered to have a high risk for onset and exacerbation of IE.

Diagnosing IE is challenging; several investigations have been performed to delineate the lesions, as found in our literature review. Enhanced-CT imaging is a useful option for estimating abdominal disorders because of its ability to detect bowel dilatation, thickened intestinal wall, and intestinal blood flow. Strictures with intestinal wall thickness in the ileum are suggested to be typical findings of IE. The average length of the stricture was $7.4 \mathrm{~cm}$, which is longer than that in other causes of intestinal obstruction, such as adenocarcinoma and Crohn's disease [13]. Enhanced CT could not be performed in the current case due to severe renal failure; however, plain CT findings and contrast medium from an ileus tube revealed a typical longitudinal stricture for IE. Angiography is also an effective examination method for assessing altered vascular conditions. Because of renal failure, we also avoided angiography that enables us to describe blood vessel structures [2]. Endoscopic examination, including single or double balloon-assisted endoscopy $[1,3,5]$ and capsule endoscopy [4], provides information on the mucosal surface and luminal condition and allows physicians to obtain specimens from the intestinal mucosa [5]. Although endoscopy is a considerably effective examination to estimate the cause of small bowel obstruction, we did not perform endoscopy because of the patient's underlying severe systemic conditions. 


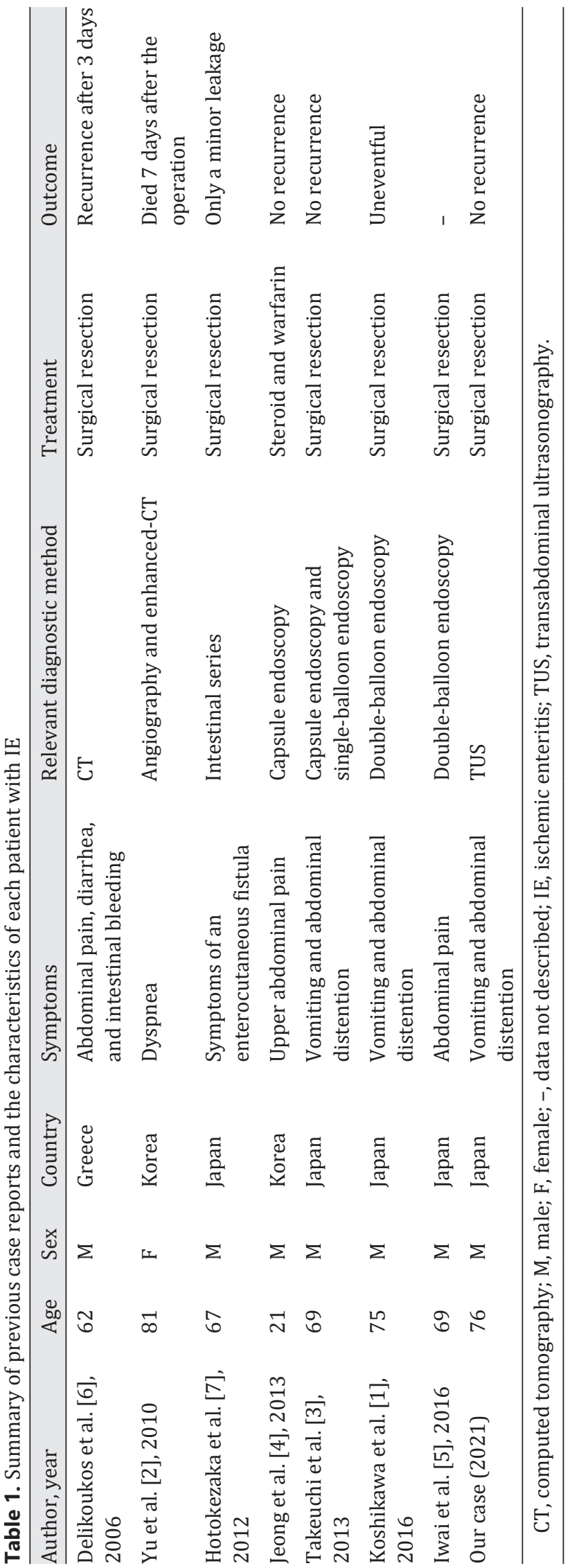




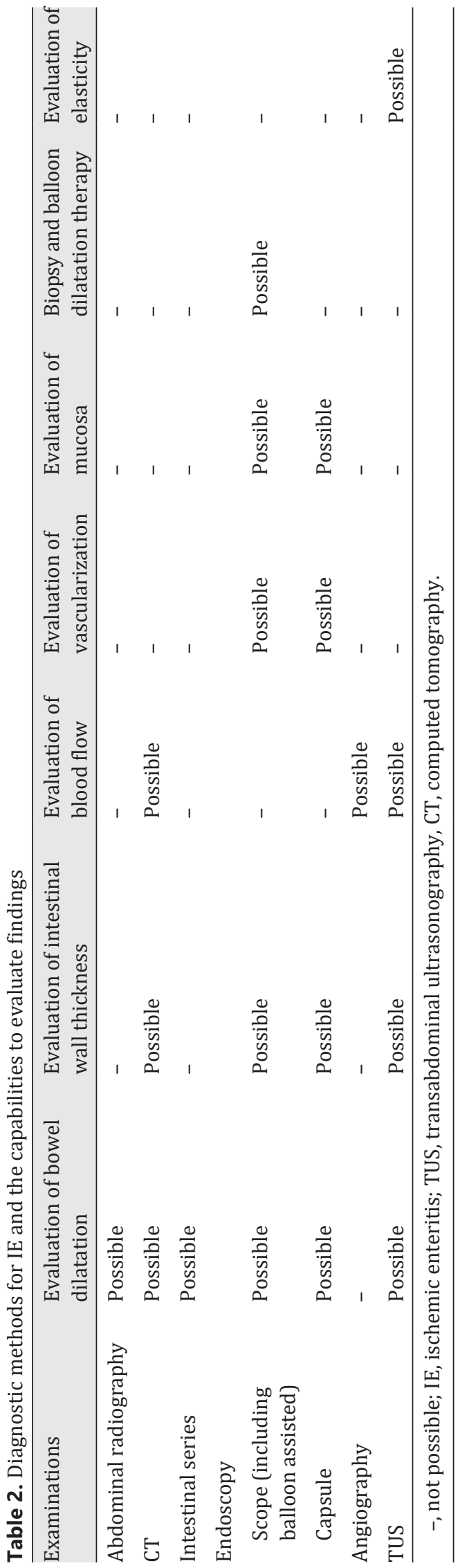


To delineate the stenotic site noninvasively, we carried out TUS and elastography techniques. TUS has been reported to be an effective option for diagnosing small intestinal disorders [14]. In our case, a 7-cm stenotic site with a thickened small intestinal wall at the end of the ileum was observed using TUS. The lesion was nonsegmental and did not show hypervascularization. The findings were neither consistent with a tumor, which exhibits short localized thickening of the wall without stratification, nor with inflammatory bowel disease, which demonstrates segmental wall thickening with focal disappearance of its stratification [15]. Moreover, using contrast-enhanced ultrasonography imaging, we were able to rule out complete intestinal ischemia. Comprehensive evaluation of the contrast-enhanced and noncontrast-enhanced ultrasonographic findings made it possible to rule out small intestinal carcinoma, which is known to demonstrate enhanced blood perfusion and hypervascularization [16]. Elastography is an emerging evaluation method for elasticity and utilizes a sonographic technique by which the relative elasticity of the lesion site can be assessed compared to the elasticity of the other tissues [17]. While this method has primarily been adopted to evaluate mammary neoplasms and fibrotic alterations of liver tissue, it has also been reported to be a useful option to assess gastrointestinal disorders such as Crohn's disease and colorectal cancer [18]. In this case, the stenotic lesion was detected to be more elastic than the normal small intestine by using strain ratio, which displayed the ratio of elasticity on the lesion to normal intestine. Further, the strain ratio was much lower than that of adenocarcinoma [19]. Based on the findings, we clinically diagnosed our patient with fibrous stenosis resulting from IE. The sonographic findings and diagnoses were consistent with the pathological diagnosis in this case.

Combining past case reports published in English, we summarized the diagnostic methods for IE with their potential targets (Table 2). Various diagnostic options were used depending on the patient's general condition, access to equipment, and technical issues. Compared to other examinations, TUS can be used to assess a broader range of information, including tissue elasticity, without any radiation exposure. Furthermore, real-time assessment can be performed noninvasively using TUS, and its cost-effective and noninvasive features allow for the examination to be performed repeatedly.

Although conservative treatment and balloon dilatation can partially alleviate symptoms [5], surgical resection is needed in most IE cases, especially in those with fibrous stenosis [1]. This clinical course is not consistent with that of ischemic colitis, for which surgical options are rarely performed [20]. We also resected the stenotic lesion in our case based on the known clinical course of IE. An additional advantage of surgical resection in addition to symptom improvement is that the entire intestinal layer can be pathologically evaluated. The histological characteristics of IE include the following findings [3]: variable ulcer depth with mostly UI (ulcer index)-II or UI-III ulceration, ulcer bases lined with vascular-rich granulation tissue; severe fibrosis within the submucosal layers, severe inflammatory cell infiltration, and hemosiderin-laden macrophages spread throughout the thickened intestinal wall. In our case, the histological findings largely matched the typical features of IE.

In conclusion, fibrotic stenosis with IE is a critical differential diagnosis of small bowel obstruction. Older subjects with cardiovascular complications and severe general conditions have a high risk of developing IE. TUS can be a harmless and highly beneficial diagnostic method for IE, especially in patients with unstable systemic conditions, including severe renal failure.

\section{Acknowledgements}

We would like to express our gratitude to the patient and to Editage (www.editage.com) for English language editing.

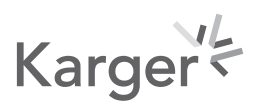




\section{Statement of Ethics}

We reported this case in compliance with the principles of the Declaration of Helsinki. This case was reviewed and approved by the institutional Ethics Committee of Kawasaki Medical School. Approval number was 2020-1528. Written informed consent was obtained from the patient for publication of this case report and any accompanying images.

\section{Conflict of Interest Statement}

The authors have no conflicts of interest to declare.

\section{Funding Sources}

The authors disclose no sponsorship or funding arrangements relating to their research.

\section{Author Contributions}

Conceptualization: N.M., K.H. Data curation: R.K., N.M., A.S., J.N., K.K., M.M., H.F., Y.M. Formal analysis: R.K. Investigation: R.K., N.M., M.A. Methodology: N.M. Project administration: R.K., N.M. Resources: R.K., N.M., J.N., K.K., M.M., H.F., Y.M., T.K., T.Y., Y.N. Software: R.K. Supervision: N.M., H.K., K.H. Validation: N.M. Visualization: R.K., N.M. Writing - original draft: R.K. Writing - review and editing: N.M., H.K. Approval of final manuscript: all the authors.

\section{References}

1 Koshikawa Y, Nakase H, Matsuura M, Yoshino T, Honzawa Y, Minami N, et al. Ischemic enteritis with intestinal stenosis. Intest Res. 2016 Jan;14(1):89-95.

2 Yu J, Kim B, Chung S, Park CW, Chang YS. Ischaemic enteritis in a patient with chronic renal failure: diagnosis and management decisions. BMJ Case Rep. 2010.

3 Takeuchi N, Naba K. Small intestinal obstruction resulting from ischemic enteritis: a case report. Clin J Gastroenterol. 2013 Aug;6(4):281-6.

4 Jeong WS, Song HJ, Na SY, Boo SJ, Kim HU, Kim J, et al. Acute extensive ischemic enteritis in a young man diagnosed with wireless capsule endoscopy: a case report. Korean J Gastroenterol. 2013 Mar;61(3):160-5.

5 Iwai N, Handa O, Naito Y, Dohi O, Okayama T, Yoshida N, et al. Stenotic ischemic enteritis with concomitant hepatic portal venous gas and pneumatosis cystoides intestinalis. Intern Med. 2018 Jul;57(14):1995-9.

6 Delikoukos S, Christodoulidis G, Zacharoulis D, Poultsidi A, Hatzitheofilou C. Multiple small bowel ruptures due to ischemic enteritis: a case report. World J Gastroenterol. 2006 Jul;12(26):4262-3.

7 Hotokezaka M, Mibu R, Maehara R, Tanaka M, Chijiiwa K, Fujino M, et al. Side-to-side isoperistaltic strictureplasty for chronic ischemic enteritis: report of a case. Surg Today. 2012 Jan;42(1):80-3.

8 Vaziri ND, Dure-Smith B, Miller R, Mirahmadi MK. Pathology of gastrointestinal tract in chronic hemodialysis patients: an autopsy study of 78 cases. Am J Gastroenterol. 1985 Aug;80(8):608-11.

9 Brener ZZ, Bergman M, Ohm HK, Winchester JF. Acute non-occlusive mesenteric ischemia of the small bowel in a patient started on hemodialysis: a case report. Cases J. 2008 Oct;1(1):217.

10 Garimella PS, Hirsch AT. Peripheral artery disease and chronic kidney disease: clinical synergy to improve outcomes. Adv Chronic Kidney Dis. 2014 Nov;21(6):460-71.

11 Park CJ, Jang MK, Shin WG, Kim HS, Kim HS, Lee KS, et al. Can we predict the development of ischemic colitis among patients with lower abdominal pain? Dis Colon Rectum. 2007 Feb;50(2):232-8.

12 Choi SR, Jee SR, Song GA, Park SJ, Lee JH, Song CS, et al. Predictive factors for severe outcomes in ischemic colitis. Gut Liver. 2015 Nov;9(6):761-6.

13 Kim JS, Kim HJ, Hong SM, Park SH, Lee JS, Kim AY, et al. Post-ischemic bowel stricture: CT features in eight cases. Korean J Radiol. 2017 Nov-Dec;18(6):936-45.

14 Kralik R, Trnovsky P, Kopáčová M. Transabdominal ultrasonography of the small bowel. Gastroenterol Res Pract. 2013;2013:896704. 
15 Kunihiro K, Hata J, Haruma K, Manabe N, Tanaka S, Chayama K. Sonographic detection of longitudinal ulcers in Crohn disease. Scand J Gastroenterol. 2004 Apr;39(4):322-6.

16 Onji K, Yoshida S, Tanaka S, Takemura Y, Oka S, Yoshihara M, et al. Microvascular structure and perfusion imaging of colon cancer by means of contrast-enhanced ultrasonography. Abdom Imaging. 2012 Apr;37(2): 297-303.

17 Dietrich CF, Barr RG, Farrokh A, Dighe M, Hocke M, Jenssen C, et al. Strain elastography - how to do it? Ultrasound Int Open. 2017 Sep;3(4):E137-49.

18 Giannetti A, Matergi M, Biscontri M, Tedone F, Falconi L, Giovannelli L, et al. Strain elastography in Crohn's disease: the role of visual observation and semiquantitative parameters. J Ultrasound. 2019 Jun;22(2):22739.

19 Waage JE, Leh S, Røsler C, Pfeffer F, Bach SP, Havre RF, et al. Endorectal ultrasonography, strain elastography and MRI differentiation of rectal adenomas and adenocarcinomas. Colorectal Dis. 2015 Feb;17(2):124-31.

20 O'Neill S, Yalamarthi S. Systematic review of the management of ischaemic colitis. Colorectal Dis. 2012 Nov; 14(11):e751-63. 